\title{
Uniformity is Weaker than Semi-Uniformity for Some Membrane Systems
}

\author{
Niall Murphy*† \\ Microsoft Research, Cambridge, CB1 $2 F B, U K$ \\ a-rimurp@micmsoft.com
}

Damien Woods ${ }^{+}$

California Institute of Technology, Pasadena, CA 91125, USA

woods(a)callech.edu

\begin{abstract}
We investigate computing models that are presented as families of finite computing devices with a uniformity condition on the entire family. Examples of such models include Boolean circuits, membrane systems, DNA computers, chemical reaction networks and tile assembly systems, and there are many others. However, in such models there are actually two distinet kinds of uniformity condition. The first is the most common and well-understood, where each input length is mapped to a single computing device (e.g. a Boolean circuit) that computes on the finite set of inputs of that length. The second, called semi-uniformity, is where each input is mapped to a computing device for that input (e.g. a circuit with the input encoded as constants). The former notion is well-known and used in Boolcan circuit complexity, while the latter notion is frequently found in literature on nature-inspired computation from the past 20 years or so.

Are these two notions distinct? For many models it has been found that these notions are in fact the same, in the sense that the choice of uniformity or semi-uniformity leads to characterisations of the same complexity classes. In other related work, we showed that these notions are actually distinct for certain classes of Boolean circuits. Here, we give analogous results for membrane systems by showing that certain classes of uniform membrane systems are strictly weaker than the analogous semi-uniform classes. This solves a known open problem in the theory of membrane systems. We then go on to present results towards characterising the power of these semi-uniform and uniform membrane models in terms of NL and languages reducible to the unary languages in NL, respectively.
\end{abstract}

Keywords: membranes systems; computational complexity; uniform families; semi-uniform familics; tally languages

\footnotetext{
${ }^{*}$ Address for correspondence: Microsoft Research, Cambridge, CB1 2FB, UK.

${ }^{\dagger}$ Niall Murphy was supported by a PICATA Young Doctors fellowship from CEI Campus Moncloa, UCM-UPM, Madrid, Spain and an Embark Fellowship from the Irish Research Council for Science, Engineering and Technology.

${ }^{\ddagger}$ Damien Woods is supported by the USA National Science Foundation under grants 0832824 \& 1317694 (The Molecular Programming Project), CCF-1219274, and CCF-1162589.
} 


\section{Introduction}

Many of the early DNA computing algorithms $[22,28,29,39]$ involved mapping an instance of an NPhard problem (such as Maximal Clique) to a set of DNA strands and lab protocols, and then using wellknown biomolecular techniques to solve the problem. To assert generality for such an algorithm one would define a mapping from arbitrary problem instances to sets of DNA polymers and experimental protocols. In order to claim that this mapping is not doing the cssential computation, it would have to be easily computable (for example, logspace computable). Circuit uniformity (introduced by Borodin [11]) provides a well-established framework where we map each input length $n \in \mathbb{N}$ to a circuit $C_{n} \in \mathfrak{C}$, with a suitably simple mapping. Ilowever, some of the DNA computing algorithms cited above do something different, they map an instance $x$ of the problem to a computing device $C_{x}$ that is unique to that inpul (via a suitably simple cncoding function). This latter notion is called semi-uniformity [48, 45], and in fact quite a number of nature-inspired computational models use semi-unilormity. This raises the immediate question of whether the notions of uniformity and semi-uniformity are computationally equivalent. We investigate this question in the field ol'membrane computing or P-systems [48, 43]. This is a branch of natural computing which explores the power of computational models that are inspired by the structure and function of living cells.

It has been shown in a number of models that whether one chooses to use uniformity or semiuniformity does not allect the power of the model. However, our main result shows that uniformity is computationally strictly weaker than semi-uniformity for a number of classes of membrane systems. Specifically, we prove that choosing one notion over another in this setting gives characterisations of complexity classes that are known to be distinct. The uniform versus semi-uniform question that we address has been stated as Open Problem C in [49].

Why is this result surprising? We know that the class of problems solved by a uniform family of devices is contained in the analogous semi-uniform class, since the former is a restriction of the latter. However, it is also the case that in almost all membrane system models studied to date, the classes of problems solved by semi-uniform and uniform families turned out to be equal, see, e.g., $[4,34,55]$. Specifically, if we want to solve some problem, by specifying a family of membrane systems (or some other model), it is often much casicr to first use the more gencral notion of scmi-uniformity, and then subsequently try to find a uniform solution. In almost all cases where a polynomial time semi-uniform family of membrane systems was given for some problem [3, 45, 55], at a later point a uniform version of the same result was published $[2,4,45]$. Here we prove that this improvement is not always possible.

We go on to give a number of other results that tease out the computational power of semi-uniform and uniform families of membrane systems.

Our main result proves something general about uniform and semi-uniform families of finite devices that is independent of particular models and formalisms. Our techniques can be applied to other computational models besides membrane systems and we have demonstrated this by showing similar results for Boolean circuits [38]. Indeed, a number of other models explicitly, or implicitly, use notions of uniformity and semi-uniformity. Models presented as uniform families of devices include membrane systems and Boolean circuits as noted above, as well as DNA computers $[1,9,53,50,8]$, chemical reaction networks $[17,16,57,58]$, neural networks [42] and other models studied in computational complexity theory. Besides membrane systems, a surprising number of models, including some just mentioned, are presented as semi-uniform families of devices, including DNA computers [28, 29], chemical reaction networks $[17,57]$, the abstract tile asscmbly model $[51,54]$, the nubots model of active molecular 
sclf-assembly and robotics $[60,15]$, and an insertion-based polymer model $[18,30]$. Uniform and scmiuniform lamilies of devices are both natural ways to present a model of computation and elucidating the distinction between them seems a worthy goal.

Furthermore, although we do not formally show it, our results hold for a version of the stochastic chemical reaction network model [52] that meets our definitions for membrane systems and in particular where there arc familics of networks deciding languages and unimolecular reactions only (in the model there are discrete natural number molecular counts and all reactions are of the form $A \rightarrow \mathcal{M}$, where $\mathcal{M}$ is a mutliset of molecular species). Interestingly, these results also hold if we generalise this model to use maximally parallel synchronous reaction updates. This shows that adding the seemingly strong and unrealistic ability of maximal parallelism in this context conveys no extra power to the model (despite the fact that it does increase the power of more general, bimolecular for example, chemical reaction network models).

Our main result is of importance to work on models of computation and natural computing since it highlights that the (seemingly harmless) choice between uniformity and semi-uniformity in these models may lead to drastic changes in computational power. How drastic? Roughly speaking, we find that the semi-unilorm models studied here characterise the class $\mathrm{NL}$, while the analogous uniform models have power comparable to, or more formally reducible to, the unary languages in NL. Our work here and on Boolcan circuits suggests that this question should be asked of other computational models.

\subsection{Overview of results}

Roughly speaking, a membrane system consists of a membrane-bound compartment that contains other (possibly nested) membrane-bound compartments that in turn contain objects that interact with each other and with membranes to carry out a computation. A family, or set, of recogniser membrane systems decides a language $L$. Families can be uniform or semi-uniform. For a uniform family there is an associated pair of functions $(f, e)$, where $f$ maps a binary input word $x$, of length $n$, to a membrane system $\Pi_{n}$ that may be used to process any word of length $n$, and $e$ encodes $x$ as a multiset of input objects to $\Pi_{n}$ (for each of the $2^{n}$ words of length $n \in \mathbb{N}$ we have a single membrane system $\Pi_{n}$ ). For a semi-uniform family, a single function $h$ maps the input word $x$ to a membrane system $\Pi_{2}$ (for each word we have a membrane system). In cither case, rules are applicd to objects in the membrane system until it produces special object(s) indicating that $x$ is accepted or rejected. Of course the encoding functions $f, e, h$ should be suitably simple so that the membrane system, and not the encoding functions, are doing the intercsting work. In this paper we use $\mathrm{FAC}^{0}$ uniformity and scmi-uniformity, that is, the functions $f, t, h$ are in $\mathrm{FAC}^{0}$, the class of functions computed by uniform constant depth polynomial size Boolcan circuits; this is a class of fairly simple problems and is mostly known for what it does not contain.

In Section 3 we give our main result, that uniform families of active membrane systems without charges and dissolution (denoted $\mathcal{A} M_{-d}^{0}$ ) that run in polynomial time are strictly wcakcr than their semi-uniform counterpart. We prove this by showing that these uniform families solve no more than non-uniform- $A C^{0}$, a class that does not even contain Parity (the set of words over $\{0,1\}$ with an odd number of 1s). The analogous scmi-uniform systems can indecd solve Parity and do much clse besides. In fact, for two out of three models that we consider, the semi-uniform families exactly characterise NL. This is shown in Section 4 and illustrated as Theorem 4.1 at the top of Figure 1.

This leaves the question: what is the exact power of uniform families of $\mathcal{A} \mathcal{M}_{-d}^{0}$ systems? In previous papers, where more powerful membrane systems and complexity classes are studied, c.g. [2,3,4], model 


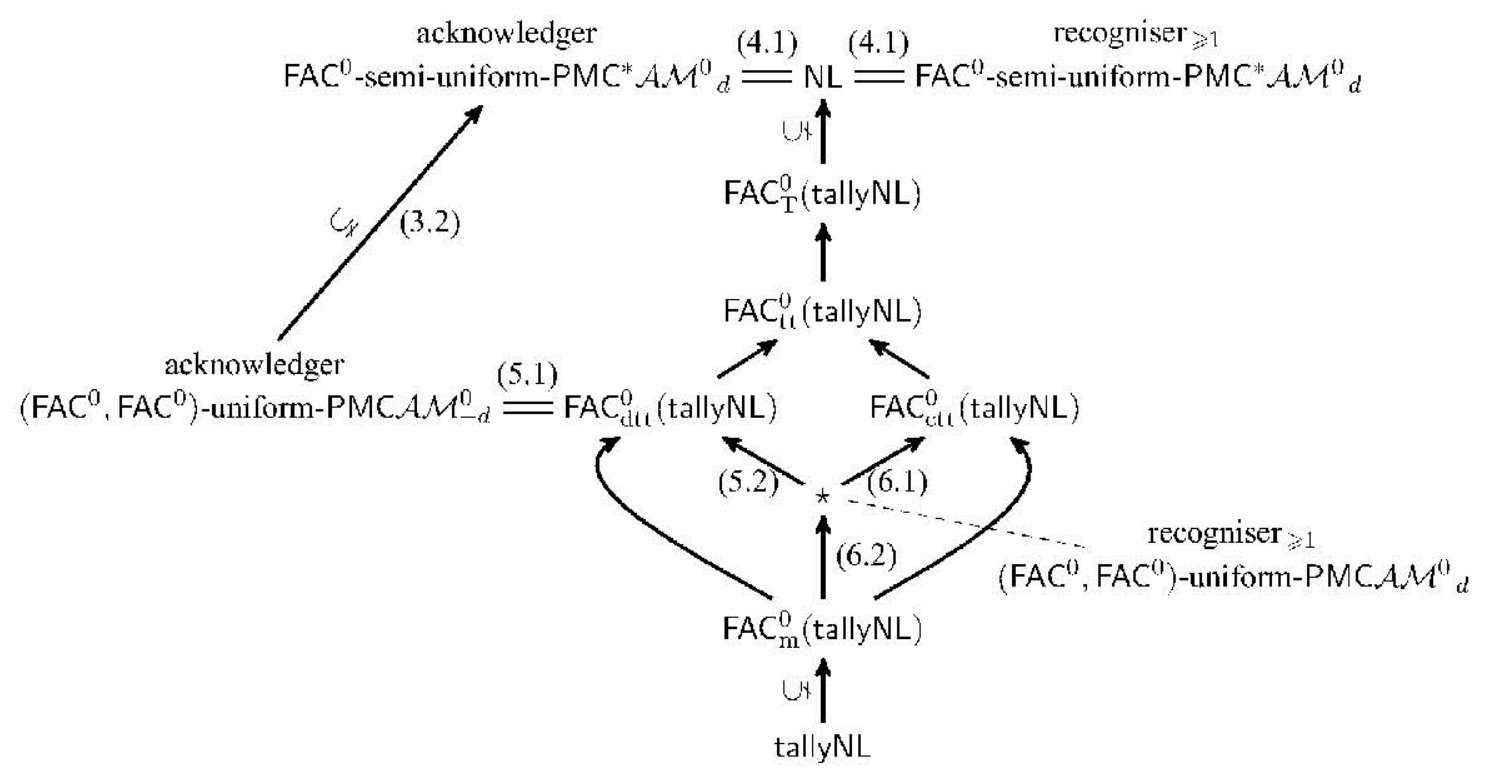

Figure 1. Summary of results. Numerical labels refer to theorems which are proved in this paper, and symbols are used to show inclusion type, with an unlabelled arrow denoting $\subseteq$. The figure shows the relationship between NL, tallyNL (the set of unary languages decided in non-deterministic logspace), and a number of classes $F A C_{r}^{(1)}$ (tallyNL) of languages that are reducible to tallyNL by various types of $F A C^{0}$ computable reductions $r$. The star $(\star)$ indicates the class labeled by the dashed linc. Sec [38] for proofs of inclusions that do not have numerieal labels and for more on classes reducible to tallyNL.

definitional choices were not so important. In our setting, definitional details such as the choice of uniformity condition and the particular kinds of acceptance modes allowed for such recogniser membrane systems lead to seemingly different results and some open questions as we now describe.

We give results for three variants on the definition of recogniser membrane system. The most powerful are acknowledger membrane systems, where an accepting computation should produce one or more yes objects, and a rejecting computation should produce zero yes objects. In Section 5 we give an exact characterisation of uniform families of acknowledger $\mathcal{A M}^{0}{ }_{d}$ membrane systems. It turns out that they decide exactly those languages that are $F A C^{0}$ disjunctive truth-table reducible to the unary languages in NL (called tallyNL). See Theorem 5.1 in Figure 1.

In Section 6 we consider recogniser $\geqslant 1$ membrane systems: a restriction of acknowledger systems where an aceepting computation produces one or more yes objects and zero no objects, and a rejecting computation produces one or more no objects and zero yes objects. We give upper and lower-bounds, in terms of classes reducible to tallyNL, for uniform families of recogniser $\geqslant 1 \mathcal{A M}_{-d}^{0}$ systems. In Figure 1, two upper bounds are illustrated as Theorems 5.2 and 6.1, and a lower bound as Theorem 6.2.

The more standard, uniform recogniser systems, are a restriction of recogniser $\geqslant 1$ membrane systems and are defined so that an accepting computation should produce a single yes object and zero no objects, and a rejecting computation should produce a single no object and zero yes objects. As noted above, our results (Figure 1, Theorem 3.2) show that these uniform recogniser systems are strictly weaker than semi-uniform recogniser systems in our setting. We do not give a tight characterisation for the power of uniform recogniser systems, but discuss this as an open problem in Section 7. 
We note that there is a previous $P$ characterisation for both uniform and semi-uniform families of active membrane systems wilhoul charges and dissolution [21]: the same systems as we use here, but under much more general uniformity conditions, namely polynomial time, or $\mathrm{P}$, uniformily. In that work the authors are motivated by the relationship with classes above $\mathrm{P}$ and so it is sufficient in their work to use $P$ uniformily. When using signilicantly tighter unilormity conditions (e.g. FAC ${ }^{0}$ ), such polynomialtime encoding functions for uniform and semi-uniform families can be seen to be stronger than the membrane systems themselves [37] (assuming NL $\subsetneq$ P). In this paper we use FAC ${ }^{0}$ uniformity which is weak enough to expose the underlying power of certain, suitably weak, classes of active membrane systems without charges or dissolution. A number of other varieties of membrane systems (e.g. [20,40]) also claim $P$ characterisations that depend on $P$ unilormity. We leave it as a possible direction for future work to investigate these, and other, membrane systems under suitably tight notions of uniformity or semi-uniformity.

\section{Definitions}

For a function $f:\{0,1\}^{*} \rightarrow\{0,1\}^{*}$ and integers $m, n \geq 1$ let $f_{n}:\{0,1\}^{n} \mapsto\{0,1\}^{m}$ be the restriction of $f$ to domain and range consisting of strings of length $n$ and $m$ respectively. We consider only functions $f$ where for each $n$ there is an $m$ such that all length- $n$ strings in $f$ 's domain are mapped to length-m strings, thus $f=\bigcup_{n=0}^{\infty} f_{n}$. Each language $L \subseteq\{0,1\}^{*}$ has an associated total characteristic function $\chi_{L}:\{0,1\}^{*} \mapsto\{0,1\}$ defined by $\chi_{L}(w)=1$ if $w \in L$ and 0 if $w \notin L$. We say a language $L$ is decided by a Turing machine $M$ il $M$ compules the characteristic lunction $\chi_{L}$. For a string $w$, we let $|w|$ denote its length.

Let NL be the class of languages accepted by non-deterministic logarithmic-space Turing machines. Such machines have a read-only inpul tape, a write-only output tape and a read-write work tape whose length is a logarithmic function of inpul length. The class of lunctions computed by a deterministic logarithmic-space Turing machines (with an additional write-only output tape) is denoted FL.

Let tally be the set of all languages over the one-letter alphabet $\{1\}$. We define tallyNL $=$ tally $\cap N L$, i.e. the class of all tally languages and length encoded languages in NL. For more details on complexity classes and Turing machines see [41].

A circuit $C_{n}$ computes a function computes a function $f_{n}:\{0,1\}^{n} \mapsto\{0,1\}^{m}$ on a fixed number $n$ of Boolcan variables. We consider functions of an arbitrary number of variables by defining (possibly infinite) families of circuits. We say a family of circuits $\mathfrak{C}=\left\{C_{n} \mid n \in \mathbb{N}\right\}$ computes a function $f:\{0,1\}^{*} \mapsto\{0,1\}^{*}$ if for all $n \in \mathbb{N}$ and for all $u \in\{0,1\}^{n}$ circuit $C_{n}$ outputs the string $f_{n}(w)$. We say a lamily ol' circuits $\mathfrak{C}$ decides a language $L \subseteq\{0,1\}^{*}$ if lor each $w \in\{0,1\}^{n}$ circuit $C_{n} \in \mathfrak{C}$ on inpul $w$ compules $\chi_{L}$.

In a non-uniform family of circuits there is no required similarity between family members. In order to specily such a requirement we use a uniformity function that algorithmically specifies the similarity between members of a circuil lamily. Roughly speaking, a uniform circuit family $\mathfrak{C}$ is an infinite sequence of circuits with an associated function $f:\{1\}^{*} \rightarrow \mathfrak{E}$ that generates members of the family and is computable within some resource bound. For more details on Boolean circuits see [59].

When dealing with uniformity for small complexity classes one of the preferred uniformity conditions is DLOGTIME-uniformity [32]. Roughly speaking, a circuit is DLOGTIME-uniform if there is a procedure that can decide if a word is in the "connection language" of the circuit family in time lincar 
in the word length. Each word of the connection language encodes cither an input gate of the circuit, an oulput gate of the circuit, or a wire connecting the output ol" one identified gate to the input of a second identified gate. Each word also encodes, in binary, the number $n$ for this circuit. For more details on DLOGTIME unilormity see [5, 32].

The depth of a circuit is the length of the longest path from an input gate to an output gate. The size of a circuit is the number of wires it contains [5].

Non-unilorm- $\mathrm{AC}^{0}$ is the set of languages decidable by lamilies of constant-depth polynomial-size (in inpul length $n$ ) circuits with unbounded lan-in $A N^{-D}$ and $\mathrm{OR}$ gates, and NOT gates with lan-in" $1 . \mathrm{AC}^{0}$ is the set of languages decidable by constant-depth polynomial-size (in input length $n$ ) DLOGTIME-unilorm circuits with unbounded lan-in AND and OR gates, and NOT gates with lan-in 1. $F_{A C}^{0}$ is the class of functions computable by polynomial-size constant-depth DLOGTIME-uniform circuits with unbounded fan-in AND and OR gates, and NoT gates with fan-in 1.

\subsection{Reductions}

For concreteness, we explicitly define some standard types of reductions. Let $A, B \subseteq\{0,1\}^{*}$. Let $C$ be a set of lunctions (for example $\mathrm{FL}$ or $\mathrm{FAC}^{0}$ ), a lunction $f$ is $\mathrm{C}$-computable if $f \in \mathrm{C}$.

Definition 2.1. (Many-one reducible)

Set $A$ is many-one reducible to set $B$, written $A \leq_{\mathrm{m}}^{\mathrm{C}} B$, if there is a lunction $f$ that is C-computable with the property that for all $w, w \in \Lambda$, if and only if $f(w) \in B$.

The following definition of truth table reduction comes from $[10,12]$, see also $[27,47]$. The Boolcan function $\sigma$ is historically called a truth table [47].

Definition 2.2. (Truth-table reduction)

Set $A$ is $\mathrm{C}$ truth table reducible to set $B$, written $A \leq_{\mathrm{tt}}^{\mathrm{C}} B$, if there exists C-computable functions $\tau$ : $\{0,1\}^{*} \rightarrow\{0,1\}^{*} \times\{0,1\}^{*} \times \ldots$ and $\sigma:\{0,1\}^{*} \rightarrow\{0,1\}$ such that $u \in A$ if and only if $\tau(w)=$ $\left(a_{1}, \ldots, a_{\xi_{w}}\right)$ such that $\sigma\left(\chi_{B}\left(a_{1}\right), \ldots, \chi_{B}\left(a_{\xi_{w}}\right)\right)=1$, where $\chi_{B B}$ is the characteristic function of $B$.

A disjunctive truth table reduction (dit) is one where at least one string generated by $\tau(w)$ is in $B$, in other words $\sigma\left(\chi_{B}\left(a_{1}\right), \ldots, \chi_{B}\left(a_{\ell_{w}}\right)\right)=V_{1 \leq i \leq \xi_{w}} \chi_{B}\left(a_{i}\right)$. A conjunctive truth table reduction (ct1) is one where all the strings generaled by $\tau(w)$ are in $B$, in other words $\sigma\left(\chi_{B}\left(a_{1}\right), \ldots, \chi_{B}\left(a_{\ell_{w}}\right)\right)=$ $\Lambda_{1 \leq i \leq \ell_{w}} \chi_{B}\left(a_{i}\right)$.

\section{Definition 2.3. (Turing reducible)}

Set $A$ is $C$ Turing reducible to $B$, written $A \leq_{\mathrm{T}}^{\mathrm{C}} B$, if there is a Turing machine $M$, that is resourcebounded in the same way machines computing functions in $C$ are, such that $w \in A$ iff $M$ accepts $w$ with $B$ as its oracle.

The following implications follow directly from these definitions, for more details see [27].

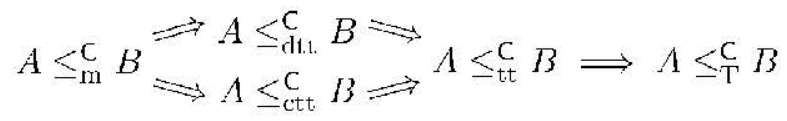

Let $F A C_{r}^{0}(C)$ be the set of all languages that are $F A C^{0}$ reducible to languages in $C$ via a reduction of some type $r \in\{m, d t t, c t t, t t, T\}$. 


\subsection{Configuration graphs}

\section{Definition 2.4. (Configuration Graph)}

Let $w \in\{0,1\}^{*}$ be the input to a halting $s(|w|)$-space bounded Turing machine $M$. The configuration graph $G_{M, u}$ of $M$ on input $w$ is an acyclic directed graph where for each potential configuration of $M$ there is a vertex that encodes it and where a potential configuration consists of an input read bit, work tape contents, input tape head position and work tape head position. The graph $G_{M \text {,wi }}$ has a directed edge from a vertex $c$ to a vertex $c^{\prime}$ if the configuration encoded by $c^{\prime}$ can be reached from the configuration encoded by $c$ in one step via $M$ 's transition function.

A configuration graph $C_{M, w}$ has the property that there is a directed path from the vertex $\epsilon_{s}$ representing the initial configuration, to the accept vertex $c_{c}$ if an only if $M$ accepts input $w$. Also, we consider only space bounded Turing machines that do not repeat a conliguration (i.e. loop), hence we define configuration graphs to be acyclic which will be a useful property later on. We are interested in $\mathcal{O}(\log |w|)$ space bounded Turing machines, whose configuration graphs are of size (number of vertices) $\mathcal{O}\left(|w|^{2}|Q|\right)$. Lemma 2.5 follows from Theorem 3.16 in [25].

Lemma 2.5. Given the binary encoding of a Turing machine $M$, which has state set $Q$ and an FAC computable space bound $\mathcal{O}(\log |w|)$, and given an inpul $w$, the configuration graph $C_{M, w}$, of size $\mathcal{O}\left(|w|^{2}|Q|\right)$, is computable in DLOGTIME-uniform-FAC .

\subsection{Membrane systems}

In this section we define the specific variant of membrane systems we use in this paper. We also define recognizer membrane systems, uniform families and some complexity classes. These definitions are based on those from the literature [31, 44].

In this paper the term membrane systems and the notation $\mathcal{A M}_{-d}^{0}$ refer to active membrane systems without charges and without dissolution rules $[21,44]$.

Let MS $(O)$ represent the set of all multiscts over the clements of the finite set $O$.

Definition 2.6. A membrane system of type $\mathcal{A} M_{-l l}^{0}$ is a tuple $\Pi=(O, \mu, M, H, \Lambda, R)$ where:

- $O$ is the alphabet of objects (or the set of object types);

- $\mu=\left(V_{\mu}, F_{\mu}, e n v\right)$ is a rooted tree representing the membrane structure. $V_{\mu} \subsetneq \mathbb{N}$ is the linite set of membranes. $E_{\mu} \subsetneq V_{\mu} \times V_{\mu}$ such that $(p, c) \in E_{\mu}^{\top}$ if the (parent) membrane $p$ contains the (child) membrane $c$. The root, $m u \in V_{\mu}$, of the tree is the only membrane with no parent and is called the "environment". Leaves of the tree represent "elementary membranes": i.e. membranes which contain no other membranes.

- $M: V_{\mu} \rightarrow \mathrm{MS}(O)$ map each membrane to an object multiset, defining the membrane's object contents;

- $\Lambda: V_{\mu} \rightarrow H$ is an injective mapping of membranes to $H$, the finite set of membrane labels. In this work the environment membrane always has the label "env";

- $R$ is a finite set of developmental rules of the following types (where $o, u, c \in O$ and $u \in \operatorname{MS}(O)$, $h \in H)$ : 
(a) $\lceil o \rightarrow w\rceil_{h}$ (object rewriting), an object $o$ in a membrane with label $h$ is replaced by a multiset of objects $w$.

(b) $o[\rceil_{h} \rightarrow[u\rceil_{h}$ (communication in), an object $o$ in a membrane with a child membrane with label $h$ is moved into the child membrane and modified to become $u$.

(c) $[o]_{h} \rightarrow[]_{h} u$ (communication out), an object $o$ in a membrane with label $h$ is moved into the parent membrane and modified to become $u$.

(e) $[o]_{h} \rightarrow[u]_{h}[v]_{h}$ (elementary membrane division), an elementary membrane with label $h$ containing object $o$ is duplicated, in one copy $o$ is replaced by $u$ while in the other copy it is replaced by $v$.

The environment membrane cannot divide nor communicate out objects. ${ }^{1}$

The missing (d) rule is the dissolution rule which we do not consider in this paper. Active membrane systcms may also have non-elementary membrane division rulcs [44]. That is, membrancs with child membranes may also divide. For the kinds of membrane systems we consider in this paper the inclusion or omission of non-elementary division rules does not affect the results $[21,37]$.

A configuration $\mathcal{C}$ of a membrane system is a tuple $\left(\mu=\left(V_{\mu}, E_{\mu}, t m u\right), M, \Lambda\right)$ whose elements are defined in Definition 2.6 (with the exception that $\Lambda$ may be non-injective).

A permissible encoding of a membrane system $\langle 1 \Gamma\rangle$, or ol'a configuration $\langle\mathcal{C}\rangle$, encodes all multisets in a unary manner. For example, a multiset is encoded in the format $[a, a, a, b, b]$, rather than in the shorter format $a^{3} b^{2}$. Likewise, the membrane structure should be encoded such that each membrane child-parent relation is written explicitly.

A configuration $\mathcal{C}_{i}$ transitions to configuration $\mathcal{C}_{i+1}$ by the application of a multiset of rules $\mathcal{R}$ from the set $R$. The rules are applied in a maximally parallel manner. That is, at each timestep, a multiset of applicable rules $\mathcal{R}$ is non-deterministically chosen such that (i) all rules in $\mathcal{R}$ are applicable, and (ii) there does not exist a multiset of applicable rules $\mathcal{R}^{\prime}$ such that $\mathcal{R} \subsetneq \mathcal{R}^{\prime}$. Rules are applicable in a timestep according to the following principles: Rules are applied to the most deeply nested membranes first. In each timestep, an object can be involved in at most one rule of any type. A membrane can be the subject of at most one rule of type (b), (c) or (e). If a membrane is divided (a rule of type (e)) and there are objects in this membrane which evolve via rules of type (a), then we assume that first the type (a) rules are applied, and then the division rule. All other rules are applicd non-deterministically.

A computation of a membrane system is a sequence of configurations where cach configuration transitions to the next. As noted above, at a given timestep the multiset of applicable rules is nondeterministically chosen: therefore on a given input there are multiple possible computations. In other words, membrane systems are non-deterministic. A computation that reaches a configuration where no more rules are applicable is called a halting computation.

\subsubsection{Recogniser, recogniser $\gtrless_{1}$, and acknowledger membrane systems}

For the following three definitions it is the case that the set of objects $O$ contains the special objects yes and no and that there are no rules applicable to yes or no (hence if yes or no are created, they can never be destroyed). The standard [44] definition of a recogniser membrane system is as follows.

${ }^{1}$ Definitions of active membranes often include a second container membrane that cannot dissolve called the "skin" [44], we omit this from our definitions. The proofs in this paper can be casily modified to account for a skin. 
Definition 2.7. A recogniser membrane system is a mombranc system such that all computations halt, and at the halting step (and not belore) exactly one of the objects yes $\in O$ or no $\in O$ appears in the multiset of the environment membrane.

A computation that halts with yes in the environment is referred to as an accepting computation while one with no in the environment is referred to as a rejecting computation. In this paper, and in previous work $[36,37]$, we also use the following more general systems:

Definition 2.8. A recogniser $\geqslant 1$ membrane system is a mombranc system such that all computations halt, and either (a) one or more copies of the object yes $\in O$ or (b) one or more copies of the object no $\in O$ appear in the multiset of the environment membrane, but not both.

As with recogniser membrane systems, a computation of a recogniser $\geqslant 1$ membrane system that halts with yes in the environment is referred to as an accepting computation while one with no in the environment is referred to as a rejecting computation. In this paper we also use the following systems that are more general than the two above:

Definition 2.9. Acknowledger membrane systems are systems such that all computations halt (and where one or more copies of the distinguished object yes may or may not appear in the env membrane).

We say that a computation of an acknowledger membrane system is an accepting computation if at least one yes object is present in the env membrane at the linal step. A compulation of an acknowledger membrane system is in a rejecting computation if there are zero yes objects in the env membrane at the linal step.

\subsubsection{Families of membrane systems}

There are two main notions of uniformity considered in the membrane computing literature defined as follows.

\section{Definition 2.10. (Semi-uniform families)}

A family of membrane systems systems $\Pi=\left\{\Pi_{w} \mid w \in \Sigma^{*}\right\}$ is said to be semi-uniform if there is a function $h: \Sigma^{*} \mapsto \Pi$ that maps from each input word $w$ to a description (in a permissible encoding) of a membrane system $\Pi_{w}$.

\section{Definition 2.11. (Uniform families)}

A family of membrane systems $\Pi=\left\{1 \complement_{n} \mid n \in \mathbb{N}\right\}$ is said to be uniform if there are two associated functions:

1. $f: 1^{*} \mapsto \Pi$ that maps $1^{n}$ (the unary representation of $n$ ) to the description (in a permissible encoding) of a membrane system $\Pi_{n}$ with a designated input membrane;

2. $e: \Sigma^{*} \rightarrow \mathrm{MS}(O)$ that maps a word $w \in \Sigma^{*}$ to the input multiset $e(w)$ (in a permissible encoding) where $O$ is the set of objects of $f\left(1^{n}\right), n=|w|$.

We let $\Pi_{n}(e(w))$ denote the membrane system $f\left(1^{n}\right)=\Pi_{n}$ with the multiset $e(w)$ in its designated input membranc. Notc that both $\Pi_{n}$ and $\Pi_{n}(e(w))$ are membrane systems. 
In this paper, we deal only with confluent membrane systems: in a confluent membrane system $\Pi$ all computations of 15 agree on the answer, that is, either all of 1 ['s computations are accepting (in which case $\Pi$ accepts) or else all of $\Pi$ 's computations are rejecting (in which case $\Pi$ rejects).

A semi-uniform family, $\Pi$, recognises a language $L \subseteq \Sigma^{*}$ confluently if for all $w \in \Sigma^{*}$ there exists $l \Gamma_{w} \in \Pi$ such that $w \in L$ implies that ${ } \Gamma_{w}$ accepts confluently and $w \notin L$ implies $l \Gamma_{w}$ rejects confluently. A uniform family, $\Pi$, with encoder $\theta$, recognises a language $L \subseteq \Sigma^{*}$ confluently if for all $w \in \Sigma^{*}$ there exists $\Pi_{|w|}(e(w))$ where $\Pi_{|w|} \in \Pi$ such that $w \in L$ implies that $\Pi_{|w|}(e(w))$ accepts confluently and $w \notin L$ implies $\operatorname{lI}_{|w|}(e(w))$ rejects conlluently. Such a (semi-)uniform lamilies are called a confluent families of recogniser, recogniser $\geqslant 1$, or acknowledger membrane systems.

That is, each membrane system II in a confluent family starts from a fixed initial configuration and then $\Pi$ non-deterministically chooses one from a number of valid computations. All of these valid computations give the same result: either all accepting (if $w \in L$ ) or else all rejecting (if $w \notin L$ ).

If the functions $f\left(1^{|w|}\right)$ and $e(w)$ (or respectively the single function $h(w)$ ) for a (semi-)uniform family are computable in time polynomial in $|w|$ on a Turing machine we say the family uses polynomial time (semi-)uniformity. If the uniformity functions are computable by DLOGTIME uniform constant depth circuits, that is, $f, e, h \in \mathrm{FAC}^{0}$, then the family is said to use constant depth uniformity.

In this paper we consider two classes of problems, those that can be solved by $\mathrm{FAC}^{0}$-uniform families of confluent $\mathcal{A} M_{-d}^{0}$ (active membranes without charges or dissolution rules) that run in time polynomial in $|w|$, denoted $\left(\mathrm{FAC}^{0}, \mathrm{FAC}^{0}\right)$-uniform-PMC $\mathcal{A} M_{-d}^{0}$, and $\mathrm{FAC}^{0}$-semi-uniform families of confluent $\mathcal{A M}_{-d}^{0}$ systems that run in time polynomial in $|w|$, denoted $\mathrm{FAC}^{0}$-semi-uniform-PMC ${ }^{*} \mathcal{A M}_{-}^{0}$.

\subsection{Context-freeness in membrane systems}

Lemma 2.12. Let $o$ be an object in a membrane with label $h$ in a configuration $\mathcal{C}_{i}$ of a membrane system II. Remove all other objects from $\mathcal{C}_{i}$ to get configuration $\mathcal{C}_{i}^{\natural}$. If there is a rule $r$ in $1[$ such that by applying that rule $10 o, h$ in $\mathcal{C}_{i}^{\natural}$ gives a configuration $\mathcal{C}_{i+1}^{\emptyset}$ with object $o^{\prime}$ in $h^{\prime}$, then it is the case that from configuration $\mathcal{C}_{i}$ there exists a configuration $\mathcal{C}_{i}$, reachable in a single step that contains $o^{\prime}$ in $h^{\prime}$.

\section{Proof:}

The rule $r$ is of the type (a), (b), (c) or (c) as described in Definition 2.6. It is sufficient to show that there is always at least one maximal set of rule applications for configuration $\mathcal{C}_{i}$ that creates $o^{\prime}$ in $h^{\prime}$ in $\mathcal{C}_{i+1}$.

Recall that an object in a configuration can be involved in at most one rule of any type. If the rule $r$ is of type (a), it has the form $\left[\left.0 \rightarrow \sigma^{\prime} w\right|_{b}\right.$, where $w$ is a (possibly emply) string over $O$ and it is necessarily the case that $h=h^{\prime}$ (rules of type (a) are applied within a single membrane).

Let the notation $\mathcal{C}_{i}-\{o\}$ denote the configuration $\mathcal{C}_{i}$ without the instance of the object o under consideration and consider any maximal multiset $R$ of rules that can be applied to the configuration $\mathcal{C}_{i}-\{0\}$. Also, consider the multiset of rule applications $R$ unioned with the application of the rule $\left[o \rightarrow o^{\prime} w\right]_{h}$ to our object instance $o$ in the relevant membrane with label $h$ in $\mathcal{C}_{i}$. We claim that this new multiset is a maximal multiset of rules that can be applied to $\mathcal{C}_{i}$. To see this notice that object instance $o$ has a rule being applied to it, and each object can have at most one rule applied to it, and no other objects with applicable rules are without rules because $R$ was maximal. IIence there is a maximal multiset of rule applications for $\mathcal{C}_{i}$ that applies $r$ and hence when it is applied $\mathcal{C}_{i_{1}}$ contains $o^{\prime}$ in a membrane with label $h=h^{\prime}$.

Rules of type (b), (c) and (e) involve both an object and a membrane. Consider $\mathcal{C}_{i}-\{0\}$ defined as above, and consider any maximal multiset $R$ of rule applications to $\mathcal{C}_{i}-\{o\}$. Furthermorc, if in $R$ 
there is a rule that involves the membrane with label $h$ where object instance $o$ was, then remove that rule application from $R$ to get $\hat{h}$. We claim that the multiset of rule applications $\hat{R}$, unioned together with the rule application "rule $r$ applied to our object instance $o$ contained in the membrane with label $h$ " is a maximal multiset of rule applications for $\mathcal{C}_{i}$. To see this note that (i) $r$ is now being applied to the relevant instances of $o, h$ so no other rule can be applied to that object nor to the membrane with that label, and (ii) there are no other rules that can be applied because $R$ was maximal. After the application of this maximal multiset of rules the new configuration $\mathcal{C}_{i \mid 1}$ contains $o^{\prime}$ in a membrane with label $h^{\prime}$.

The following lemma generalises Lemma 2.12 from one to multiple computation steps, and applics it to the setting of systems that recognise languages. Intuitively, it states that if a sequence of rules $r$ can be applied starting from some configuration it is not possible to prevent this from happening by adding new objects to that conliguration.

Lemma 2.13. Let $1[$ be a recogniser, recogniser $\geqslant 1$ or acknowledger membrane system. Let $o$ be an object in a membrane with label $h$ in a configuration $\mathcal{C}_{i}$ of $\Pi$. Remove all other objects from $\mathcal{C}_{i}$ to get configuration $\mathcal{C}_{i}^{\natural}$. If starting from configuration $\mathcal{C}_{i}^{\Theta}$ there is a computation that halts after $t$ steps on configuration $\mathcal{C}_{i+t}$ that contains object yes in the environment then it is the case that starting from configuration $\mathcal{C}_{i}$ there exists a halting computation with yes in the environment.

\section{Proof:}

By hypothesis we know that there is a sequence of $t$ rules $r_{1}, r_{2}, \ldots, r_{1}$ that can be applied to $\mathcal{C}_{i}^{\natural}$ to get yes in the environment. We apply Lemma $2.12 t$ times, first to configuration $\mathcal{C}_{i}$ with $r=r_{1}$, then to $\mathcal{C}_{i|1|}$ with $r=r_{2}$, and so on until we get configuration $\mathcal{C}_{i} t$ which contains yes in the environment.

If $1[$ is a recogniser system then we are done: recogniser systems produce yes in the halting step. If $\Pi$ is a recogniser $\geqslant 1$ or acknowledger membrane system we add the fact (from Section 2.3.1) that no rules can be applied to the object yes, and since there is a computation where yes is in the environment at configuration $\mathcal{C}_{i+t}$, then it remains there until the computation eventually halts.

Lemma 2.13 shows that the kind of membrane systems studied in this paper intuitively exhibit some notion of context-freeness. Essentially, there is a sense in which an object $o_{s}$ can be said to trigger a sequence of rules that eventually result in the production of object $o_{l}$ on some computation, and specifically, the production of $o_{t}$ can not be prevented by starting over with more objects (more context) in the system. Ilence, the ideas used in the proof of Lemma 2.13 justify the use of the following definition in our proofs.

\section{Definition 2.14. (Eventually evolves)}

Let $\mathcal{C}_{s}$ be a configuration of a membrane system $\Pi$, containing an object of type $o_{s}$ in a membrane labelled $h_{s}$ (along with any number of other objects and membranes). Let $\mathcal{C}_{s}^{\natural}$ denote $\mathcal{C}_{s}$ with all objects removed except one instance of $o_{s}$ in the relevant membrane with label $h$. We say that $o_{s}$ in $h_{s}$ in configuration $\mathcal{C}_{s,}$ eventually evolves on some computation path, or for short eventually evolves, object type $o_{t}$ in a membrane labelled $h_{t}$ if there is a computation (sequence of conligurations) starting from $\mathcal{C}_{s}^{\natural}$ where the final configuration in the computation has object type $o_{l}$ in a membrane labelled $h_{t}$.

Note that if $o_{s}$ in $h_{s}$ in $\mathcal{C}_{s}$ eventually evolves yes in env this means that by Lemma 2.13 there is at least one computation (sequence of configurations) that leads to a configuration with yes in env from $\mathcal{C}_{s}$. Ilowever, since membrane systems are nondeterministic, this does not necessarily happen for all computations. 


\section{Uniformity is strictly weaker than semi-uniformity}

Theorem 3.2 proves that uniform families of membrane systems are strictly weaker than semi-uniform families of the same type. The result holds for all three definitions of acknowledger, recogniser $\geqslant 1$ and recogniser membrane systems.

Lemma 3.1. $\left(F A C^{0}, F A C^{0}\right)$-uniform-PMC $A \mathcal{M}^{0}{ }_{d} \subseteq$ non-uni form- $A C^{0}$, for acknowledger, recogniser $\geqslant 1$ and recogniser membrane systems.

\section{Proof:}

Let $L \in\left(\mathrm{FAC}^{0}, \mathrm{FAC}^{0}\right)$-uniform-PMC $\mathcal{A M}{ }^{0}{ }_{d}$, and let $\Pi$ be the $\mathrm{FAC}^{0}$-uniform family of that type that decides $L$. That is, given $w \in\{0,1\}^{*}$ there is a membrane system $\mathbf{I}_{|w|} \in \Pi$ that accepts $e(w)$ ifl $w \in L$.

We now describe a non-unilorm lamily ol constant-depth circuits $\mathfrak{C}^{\mathfrak{}}=\left\{C_{n} \mid n \in \mathbb{N}\right.$ and $C_{n}$ accepts $\left.L \cap\{0,1\}^{n}\right\}$ that recognizes $L$. For any input $w \in\{0,1\}^{*}$, we claim that circuit $C_{|w|} \in \mathfrak{C}$ decides whether or not $w \in L$. The lirst constant number of layers of the circuil $C_{|w|}$ compute the input encoding function $e(w) \in \mathrm{FAC}^{0}$. This generates a polynomial (in $|w|$ ) number of binary words that encode elements from the polynomially sized object set $O$ as well as their multiplicities (in unary).

The circuit $C_{|w|}$ then converts the list of encoded $e(w)$ objects into a single binary string $\chi$ of length $|\chi|=|O|$ such that for all $i \in\{1,2, \ldots,|O|\}$, the $i$ th bit $\chi_{i}=1$ iff $o_{i} \in O$ is in $e(w)$. That is, $\chi$ is a characteristic sequence for $e(w)$, ignoring multiplicities.

For each $i$, the bit $\chi_{i}$ is wired into a unique AND gate $a_{i}$, giving a total of $|O|$ AND gates at this level. The second input to the AND gate $a_{i}$ is from a constant gate $c_{i}$, where $c_{i}=1$ if $o_{i} \in O$ in the input membrane eventually evolves (Definition 2.14) to the yes object in the env membrane and $c_{i}=0$ otherwise.

The next layer contains a single OR gate $g$ such that for cach $i$, AND gate $a_{i}$ is wired to $g$. This OR gate is the output gate of the circuit. Also wired into the OR are $|O| \times|H|$ constant gates such that gate $c_{o, h}=1$ if both (i) $o \in O$ is in membrane labelled $h \in H$ in the initial configuration of $\Pi_{|x|}$ and (ii) $O$ in $h$ cventually cvolves to yes in the env membrane, otherwise $c_{o, h}=0$.

We now argue that the above construction of $C_{|w|}$ accepts $w \in L$. Recall that $\mathrm{I}_{|w|}(e(w))$ is a confluent membrane system and so if the computation is an accepting one, then all possible computation paths arc accepting. For a computation to be accepting, a yes object must appear in the env membranc. Therefore at least one object in the initial configuration of $1 \Gamma_{|w|}(e(w))$ must eventually evolve to be a yes in the env membranc. Also $\Pi_{|w|}(e(w))$ is confluent, therefore if at least one object in the initial configuration of $1 \Gamma_{|w|}(e(w))$ eventually evolves yes in the ent membrane, the system accepts. Since the property of whether an object in some membrane eventually evolves to object yes in the enn membrane depends only on $R$ and $\mu$ in $1_{|w|}$, and hence in turn depends only on $|w|$ (by Lemma 2.13), it can be encoded (non-unilormly) in the constants $c_{i}$ in circuil $C_{|w|}$.

Suppose $\Pi_{|w|}$ accepts regardless of the input $e(x)$. In this case one of the objects, say $o$, in the initial configuration of $\mathrm{lI}_{|w|}$ will eventually evolve to yes in the env membrane. This means the relevant gate $c_{o, h}$ will be a 1 -constant gate and so the output $\mathrm{OR}$ will evaluate to 1 and so $C_{|w|}$ accepts regardless of input.

Suppose $w \in L$, therefore at least one of the objects in in $e(w)$, when placed in the input membrane of $\Pi_{|w|}$, yields a computation that ends with a configuration with object yes in membrane env. In turn this implies that at least one of the AND gates $a_{i}$ has inputs $c_{i}=1$ and $\chi_{i}=1$ and so evaluates to 1 . Finally this causes the OR to cvaluate to 1 and so $C_{|w|}$ accepts input $w$. 
Suppose $w \notin L$, in this case none of the objects in $e(w)$ will cventually cvolve to yes in the env membrane. Thus any of the $a_{i}$ AND gates that have a constant $c_{i}=1$ as inpul will have $\chi_{i}=0$ and so will evaluate to 0 . With all 0 inputs, the output $O R$ evaluates to 0 and the circuit rejects.

This circuit is of polynomial size and its depth is the sum of the depths of the FAC encoding function (which has depth $O(1)$, by definition), the depth of the circuit that converts $e(w)$ into $\chi$ (which is $O(1)$ using masking and comparison), and 2 for the final layer of AND gates and the single OR gate. Hence $\mathfrak{C}$ is a non-unilorm- $A C^{0}$ circuit family that recognizes $L$.

Theorem 3.2. $\left(\mathrm{FAC}^{0}, \mathrm{FAC}^{0}\right)$-uniform-PMC $\mathcal{A} \mathcal{M}^{0}{ }_{d} \subsetneq \mathrm{FAC}^{0}$-semi-uniform- $\mathrm{PMC}{ }^{*} \mathcal{A} \mathcal{M}^{0}{ }_{d}$, for acknowledger, recogniser $\geqslant 1$ and recogniser membrane systems.

\section{Proof:}

$(\subseteq)$ By definition, uniform families are a restriction of semi-uniform families and so $\left(\mathrm{FAC}^{0}, \mathrm{FAC}^{0}\right.$ )-uniform-PMC $\mathcal{A} \mathcal{M}^{0}{ }_{d} \subseteq \mathrm{FAC}^{0}$-semi-uniform-PMC ${ }^{*} \mathcal{A M}^{0}{ }_{d}$.

$(\neq)$ Parity $\subseteq\{0,1\}^{*}$ is the set of binary strings that contain an odd number of $1 \mathrm{~s}$. We claim that Parity $\in \mathrm{FAC}^{0}$-semi-uniform-PMC ${ }^{*} \mathcal{A M}^{0}{ }_{d}$ for recogniser systems (and hence also for acknowledger and recogniser $\geqslant 1$ membrane systems). Let $w \in\{0,1\}, n=|w|$, and let $w=w_{1}, \ldots, w_{n}$. We will define the function $h:\{0,1\} \mapsto \Pi$, where each $h(w)=1 \Gamma_{w}$ computes $\chi$ Parity $(w)$ as lollows. Each $1 \Gamma_{w}$ has a single membrane, $e n v$, the set $O$ contains $2 n+2$ objects: $O=\left\{o_{i} \mid 1 \leq i \leq n\right\} \cup\left\{e_{i} \mid 1 \leq\right.$ $i \leq n\} \cup\{$ yes,no $\}$. The initial configuration is the membrane env containing a single object $o_{1}$ in en if $w_{1}=1$ or object $e_{1}$ in en if $m_{1}=0$. The rules of $1 \mathrm{I}_{w}$ are as follows: if $w_{i}=1$ then $\left[o_{i} \rightarrow e_{i} 1\right]_{e n v},\left[e_{i} \rightarrow o_{i} \quad 1\right]_{e n v}$ and if $w_{i}=0$ then $\left[e_{i} \rightarrow e_{i \mid 1}\right]_{e n v},\left[o_{i} \rightarrow o_{i \mid 1}\right]_{e n v}$. There are also the rules $\left[\epsilon_{n} \rightarrow \text { no }\right]_{e n v}$ and $\left[o_{n} \rightarrow \text { yes }\right]_{e n v}$.

By starting with object $o_{1}$ if $u_{1}=1$, and then changing between $e_{i}$ and $o_{i}$ if $w_{i}=1$, and not changing if $u_{i}=0$ at each timestep then we ensure that the object $o_{i}$ represents "the parity of the first $i$ bits of $w$ is odd", and $e_{i}$ represents that they are even. Thus, $o_{n}$ evolves to a single yes object if there is an odd number of $1 \mathrm{~s}$ in $w$ and $e_{n}$ evolves to a single no if there is an even number of $1 \mathrm{~s}$ in $w$.

To cnd we notc that it is known [19] that Parity $\notin$ non-uniform- $A C^{()}$. Lemma 3.1 shows that $\left(F A C^{0}, F A C^{0}\right)$-uniform-PMC $A M_{-d}^{0} \subseteq$ non-uniform- $A C^{0}$, for acknowledger, recogniser $\geqslant 1$, and recogniser membrane systems.

\section{The computational power of semi-uniform families}

In prior work [37], we have shown that semi-uniform families of recogniser $\geqslant 1$ membrane systems characterise NL. We give an alternative proof here to demonstrate techniques that we will use in later sections for uniform families.

Theorem 4.1. ([37])

$\mathrm{FAC}^{0}$-semi-uniform-PMC $\mathrm{AM}_{-d}^{0}=\mathrm{NL}$, for both acknowledger and recogniser $\geqslant 1$ membrane systems.

\section{Proof:}

Lemmas 4.2 and 4.3 give the proof lor acknowledger and recogniser $\gg 1$ membrane systems.

Lemma 4.2. ([37])

$\mathrm{FAC}^{0}$-semi-uniform-PMC* $\mathrm{AM}_{-d}^{0} \subseteq \mathrm{NL}$, for acknowledger, recogniser $\geqslant 1$ and recogniser membrane systems. 


\section{Proof:}

Let $\Pi$ be a semi-uniform family of acknowledger, recogniser $\geqslant 1$ or recogniser membrane systems that recognises $L \in$ FAC $^{0}$-semi-uniform-PMC $\mathcal{A M}^{0}$ a. Let $h:\{0,1\}^{*} \rightarrow \Pi$ be the semi-uniformity function of $\Pi$, that is, on inpul $x \in\{0,1\}^{*}, \mathrm{II}_{x}=h(x)$ accepts ill $x \in L$. We present a non-deterministic logspace Turing machine $M$ that recognises $I$.

The computation of $M$ proceeds as follows: First $M$, on input $x$, non-deterministically chooses a single object from $\mathcal{C}_{1}$, the initial configuration of $\Pi_{x}$, and stores (a string representation of) the object and its containing membrane on its work tape. Then $M$ enters a loop where at each iteration it nondeterministically chooses one of the rules applicable to the object on its work tape. If the rule is of type (a) or (c) (Definition 2.6) then $M$ replaces the current object on the work tape (the membrane remains unchanged) with a non-detcrministically chosen object from the right hand side of the rulc. If the rule is of type (b) or (c) then the object on the work tape is replaced by the object on the right hand side of the rule and the membrane on the work tape is replaced by the parent (type (c)) or child membrane (type (b)) of the current membrane. If during the computation the work tape is found to store the object yes in the eme membrane then $M(x)$ halts and acepts. Otherwisc, if there are no rules applicable to the object and membrane on the work tape, and it is not yes in env, then $M(x)$ halts and rejects.

Suppose that $x \in L$ and so $\mathrm{II}_{x}=h(x)$ accepts. This implies that there is one (or more) objects in the initial configuration of $\Pi_{x}$ that will, by the application of rules to this object and its successors, become the object yes in the env membrane by the end of the computation of $\Pi_{x:}$ (this claim follows from the kind of rules we allow - they are essentially context frec - and can be formally proven using dependency graphs [21]). Indeed, this observation holds for all three kinds of membrane systems: acknowledger, recogniser $\geqslant 1$ and recogniscr. By non-deterministically choosing an object in the initial configuration, and non-deterministically choosing the rules that are applicd to this object and its successors we ensure that there is a computation of $M(x)$ for each possible sequence of rule applications of $1 I_{x}$ lor cach object in the initial configuration $\Pi_{x}$ (this follows from Lemma 2.13). Therefore at least one computation of $M(x)$ will produce the object yes in the env membranc and so $M(x)$ accepts, by confluence. That is, if $1 \mathrm{I}_{x}$ accepts then $M$ accepts on inpul $x$.

Suppose that $x \notin L$ and so $\Pi_{x}=h(x)$ rejects. This implies that there is no valid computation of $\Pi_{x}$ where an object in the initial configuration evolves to yes in the env membrane. Indeed, this observation holds for all three kinds of membrane systems: acknowledger, recogniser $\geqslant 1$ and recogniser. In this case all computation branches of $M(x)$ will reach an object to which no further rules are applicable (that is not yes) and so will halt in the rejecting statc. That is, if $\Pi_{x}$ rejects then $M$ rejects on input $x$.

To simulate the computation of $\Pi_{x}$ in logspace, $M(x)$ recomputes relevant logarithmic sized pieces of $h(x)=1 I_{x}$ via the classic technique for composing logspace algorithms (see Chapter 4.3 of [7]) each time it needs information about $\mathrm{L}_{x}$, i.e. initial configuration, rules, or membrane structure. From the statement, $h$ is computable in $\mathrm{FAC}^{0}$. This means that the number of unique objects and labels in $\mathrm{II}_{2}$ are polynomial in $n=|x|$ and so each can be uniquely identified in binary with a string of length $\log n$. $M(x)$ uses a constant number of $\log n$ sized binary strings to encode the current object and membrane, as well as some counters and temporary storage needed to re-compute $h(x)$.

Thereforc $L$ is decided by a non-deterministic logspace Turing machinc.

Lemma 4.3. ([37])

$\mathrm{NL} \subseteq \mathrm{FAC}^{0}$-semi-uniform-PMC $\mathrm{AM}^{0}{ }_{d}$, for acknowledger and recogniser ${ }_{\geqslant 1}$ membrane systems. 


\section{Proof:}

Let $L \in \mathrm{NL}$. That is, there is a non-deterministic logspace Turing machine $M$ with one or more accepting computation paths exactly for input words $x \in L \subseteq\{0,1\}^{*}$.

We show that there is an $\mathrm{FAC}^{()}$semi-uniform family of polynomial-time membrane systems $\Pi$ that recognises $L$. We now describe a function $h:\{0,1\}^{*} \rightarrow \Pi$, computable in $\mathrm{FAC}^{0}$, such that if $x \in L$ then $h(x)=\Pi_{x}$ accepts, otherwise $\Pi_{x:}$ rejects.

Consider the configuration graph $G_{M, r}$ for $M$ on input $x \in\{0,1\}^{*}$, which is FAC computable from $M$ and $x$ (see Section 2.2 and Lemma 2.5). Also consider the Turing machine $N_{M}$ (and its configuration graph $G_{N x}$ ) that on input $x$ accepts only if all computations of $M$ reject on input $x$, that is, $x \notin L . N_{M}$ uses the standard un-reachability algorithm $[24,56]$ for non-deterministic logspace.

The function $h(x)$ constructs the configuration graph $C_{M, x}$ and modifies it to produce a membrane system $\mathrm{II}_{x}$ as follows. $O$, the set of unique objects of $1 \mathrm{I}_{x}$ has an object encoding each vertex in the configuration graphs $G_{M, x}$ and $G_{N, x}$ as well as two extra objects, yes and no. The initial configuration of $\mathrm{II}_{2}$, has a single membrane labelled env that contains two objects: $c_{i}$ which encodes the initial configuration of $M(x)$; and $c_{j}$ which encodes the initial configuration of $N_{M}(x)$. The edges of the configuration graphs $G_{M, x}$ and $G_{N, n}$ are encoded as object rewriting rules in the membrane system. If vertex $u$ has $k$ edges to vertices $v_{1}, \ldots, v_{k}$ then $h(x)$ encodes all $k$ edges as a single type $(a)$ rule: $\left[u \rightarrow v_{1}, \ldots, v_{k}\right]_{e n v}$. Let vertex (object) $c_{n}$ encode the accepting configuration of the Turing machine $M$, and let $h(x)$ include the rule $\left[c_{x} \rightarrow \text { yes }\right]_{\text {env }}$. Likewise for the vertex (object) $c_{b}$ that encodes an accepting configuration of the Turing machine $N_{M}, h(x)$ includes the rule $\left[c_{b} \rightarrow \text { no }\right]_{e n v}$.

We now argue that each member $\mathrm{II}_{x}=h(x)$ of the semi-uniform family $\Pi$, accepts ill $x \in I$.

Suppose $x \in L$, therefore Turing machine $M(x)$ accepts. This implies that configuration graph $C_{M, x}$ has the property that there is a directed path from the vertex $c_{i}$ representing the initial configuration, to the accept vertex $c_{a}$. The assumption also implies that $N_{M}(x)$ must reject, and so configuration graph $G_{N, x}$ does not have a directed path from the object $c_{j}$ encoding its initial configuration $10 c_{b}$, its acecpt configuration. Since $1 \mathrm{I}_{x}=h(x)$ directly encodes the configuration graphs as objects and rules then the existence of a path from $c_{i}$ to $c_{a}$ implies that the membrane system will produce the object yes during its compulation. The absenec of a path from $c_{j}$ to $c_{b}$ implies that the membrane system will not produce the object no during its computation. Therefore $1 \mathrm{I}_{x}$ accepts if $x \in L$.

Suppose $x \notin L$, therefore no computation paths of Turing machinc $M(x)$ accept. This implies that configuration graph $C_{M, x}$ has the property that there is no directed path from the vertex $c_{2}$ representing the initial configuration, to the accept vertex $c_{a}$. The assumption also implics that $N_{M}(x)$ must accept, and so configuration graph $Q_{N, 2}$ has a directed path from the object $c_{j}$ encoding its initial configuration to $c_{b}$, its accept configuration. Since $1 \mathrm{I}_{x}=h(x)$ directly encodes the configuration graphs as objects and rules then the existence of a path from $c_{j}$ to $c_{b}$ implies that the membrane system will produce the object no during its computation. The absence of a path from $c_{i}$ to $c_{a}$ implies that the membrane system will not produce the object yes during its computation. Therefore $\Pi_{x}$ rejects if $x \notin L$.

Since cach configuration graph is acyclic and has $p(|x|)$ nodes where $p$ is some polynomial function, it follows that the membrane system itself is of polynomial size and halts in polynomial time. The conliguration graph can be compuled in $\mathrm{FAC}^{0}$ by Lemma 2.5 .

In conclusion, function $h$ defines a semi-uniform family of polynomial time $\mathcal{A} \mathcal{M}_{-d}^{0}$ recogniser $\geqslant 1$ (and so also acknowledger) membrane systems that accept the language in $L \in \mathrm{NL}$. 
Note that the above proof fails for recogniser membrane systems since if there is more than one accepting computation (or in the rejecting case, more than one rejecting computation) then multiple copies of the object yes (or no) are produced in violation of the definition of recogniser membrane systems.

\section{The computational power of uniform families of acknowledger mem- brane systems}

In this section we focus on acknowledger membrane systems (Definition 2.9) where the accepting condition is met by the presence of one or more yes object in the environment in the last step of a computation, and the absence of yes implies rejection. We give a characterisation of uniform families of acknowledger membrane systems:

Theorem 5.1. $\left(\mathrm{FAC}^{0}, \mathrm{FAC}^{0}\right)$-uniform-PMC $\mathcal{A} M_{-d}^{0}=\mathrm{FAC}_{\mathrm{dut}}^{0}$ (tallyNL), for acknowledger membrane systems.

The proof of this result is the combination of Lemmas 5.2 and 5.3. Before giving the lemmas we first introduce the following $\mathrm{FAC}^{0}$ computable functions that will be used in the prools.

Pairing function We require an injective function that pairs two binary strings into one and is extremely easy $\left(\mathrm{FAC}^{0}\right)$ to compute. We use the pairing function that interleaves the bits of two binary string arguments $a$ and $b$. For example, the binary strings $a=a_{2} a_{1} a_{0}$ and $b=b_{2} b_{1} b_{0}$ are paired as the interlcaved string $\langle a, b\rangle=b_{2} a_{2} b_{1} a_{1} b_{0} a_{0}$. The circuits for interleaving and de-interlcaving have only a single input gate layer and a single output gate layer (and so have 2 layers). The wiring between each input and output gate can be shown to be DLOGTIME-uniform.

Binary to Unary There is a constant depth circuit family where circuit $C_{n}$ takes as input some word $w \in\{0,1\}^{n}$ and outputs $1^{x}$ where $x$ is the positive integer encoded in the first $\left\lceil\log _{2} n\right\rceil$ bits of $w$ [14]. It can be shown that this circuit lamily is DLOGTIME unilorm and so this conversion from short binary strings to unary is in $\mathrm{FAC}^{0}$.

Unary to Binary There is a constant depth circuit family where circuit $C_{n}$ takes as input some word $w=0^{n-x} 1^{x}$ where $0 \leq x \leq n$, and outputs the binary encoding of $x$ [14]. It can be shown that this circuit lamily is DLOGTIME uniform and so unary to binary conversion is in FAC ${ }^{0}$.

Lemma 5.2. $\left(F A C^{0}, F A C^{0}\right)$-uniform- $P M C \mathcal{A} M_{-d}^{0} \subseteq F A C_{d u}^{0}$ (tallyNL), for acknowledger, recogniser $\geqslant 1$ and recogniser membrane systems.

\section{Proof:}

Let $L \in\left(\mathrm{FAC}^{0}, \mathrm{FAC}^{(1)}\right)$-uniform-PMCAM $\mathcal{M}_{-d}^{0}$. That is, there exist two functions $e, f \in \mathrm{FAC}^{()}$, such that $e$ maps $x \in\{0,1\}^{*}$ to a multiset of membrane system objects (the input), and $f$ maps $u \in\{1\}^{*}$ to a membranc system, $f\left(1^{|x|}\right)=\Pi_{|x|} \in \Pi$, such that $\Pi_{|x|}$ accepts input $e(x)$ iff $x \in L$.

We claim that $L$ is $\operatorname{FAC}^{0}$ disjunctive reducible to a unary language $T$, where $T$ is decided by a non-detcrministic logspace Turing machine $\mathcal{T}$. 
Let $T$ be the set of words of the form $1^{\langle o,|x|\rangle}$ where, for all $|x| \in \mathbb{N}$ and then for all $a \in O_{|x|}$, membrane system $\mathrm{I}_{|x|} \in \Pi$ accepts if object $\sigma$ in the input membrane eventually evolves to the object yes in the ene membrane (where $O_{|x|}$ is the set of objects of $\Pi_{|x|}$, where both $x$ and $o$ are encoded in binary, $\langle\cdot$,$\rangle is the binary interleaving function defined at the start of Section 5$, and $1^{b}$ denotes the unary word over $\{1\}$ of length $b$ for a binary number $b$ ). Turing machine $\mathcal{T}$ decides words in $T$ by first converting the input word to binary and then reversing the pairing function to find $o_{i}$ and $|x|$. $\mathcal{T}$ then proceeds by simulating $1 \Gamma_{|x|}$ in non-deterministic logspace using a similar method as described in Lemma 4.2, that is, by storing a constant number of objects and membranes on its work tape and recomputing $f\left(1^{|x|}\right)$ as needed (the main difference is that $\mathcal{T}$ uses $o_{i}$ as its starting object instead of non-deterministically choosing onc). As in Lemma 4.2, $\mathcal{T}$ accepts if there cxists a valid computation in $\Pi_{|x|}$ where $o_{i}$ in the input membrane becomes yes in the env membrane. $\mathcal{T}$ rejects if there are no valid computations that lead to a yes object in the env membrane. Therefore $T$ is a tally language decided by a non-deterministic logspace Turing machine and so $T \in$ tallyNL.

We now define the function $\tau \in \mathrm{FAC}^{0}$, that maps from $\{0,1\}^{*}$ to the set of tuples of unary words, and later prove that if $x \in L$ then $\tau(x) \cap T \neq \emptyset$, otherwise if $x \notin L$ then $\tau(x)\urcorner T=\emptyset$. Let $\tau(x)=\left(u_{1}, \ldots, u_{q(|x|)}\right)$, where $q(|x|)$ is the number of object types $o_{i}$ in $e(x)$, and $u_{i}=1^{\left\langle u_{i}|| x \mid\right\rangle}$. Note that the set of unique words in $\tau(x)$ is a bijection onto the set of objects $e(x)$ so $q(|x|)$ is polynomial in $|x|$. Since $e$, the pairing linction, binary-unary conversions, as well as calculation of $q(|x|)$ are in $\mathrm{FAC}^{0}$, it is not difficult to see that $\tau \in \mathrm{FAC}^{0}$.

We now prove that $\tau$ is a disjunctive reduction from $L$ to $T$. Suppose $x \in L$, this implies that at least one of the objects in $\epsilon^{\prime}(x)$, when placed in the input membrane of $1 \Gamma_{|x|}$ evolves to a yes object in the env membrane by the end of the computation of $1 \Gamma_{|x|}$. Then, by the definition of $\tau$, if $x \in L$ then $\exists o \in \tau(x)$ such that $o \in T$.

Let $x \notin L$, this implies none of the objects in $e(x)$, when placed in the input membrane of $\Pi_{|x|}$, evolve to a yes object in the env membrane by the end of the computation of $\Pi_{|x|}$. Then, by the definition of $\tau$, if $x \notin L$ then $\nexists o \in \tau(x)$ such that $o \notin T$.

Lemma 5.3. $F A C_{\mathrm{d} t u}^{0}$ (tallyNL $) \subseteq\left(F A C^{0}, F A C^{0}\right)$-uniform-PMC $\mathcal{A M}_{-d}^{0}$, for acknowledger membrane systems.

\section{Proof:}

Let $L \in \mathrm{FAC}_{\mathrm{du}}^{0}$ (tallyNL). That is, there exists a unary language $T \subseteq\{1\}^{*}$ that is recognised by a non-deterministic logspace Turing machine $\mathcal{T}$, and a function $r \in \operatorname{FAC}^{0}$ that maps $x \in\{0,1\}^{*}$ to a set of unary words such that $r(x) \cap T \neq \emptyset$ if $x \in L$, and $r(x)\urcorner T=\emptyset$ otherwise. Let $q^{\prime}(|x|)=$ $\max \left(\left\{\max (|r(w)|) \mid w \in\{0,1\}^{|x|}\right\}\right)$, that is, the length of largest word produced by $r$ on any input of length $|x|$. Note that $q^{\prime}(|x|)$ is computable by $f$ since $r \in \mathrm{FAC}^{0}$.

We present an $\mathrm{FAC}^{0}$ uniform polynomial-time $\mathcal{A M}_{-d}^{0}$ membrane family $\Pi$ that recognises $L$. The family is composed of two functions: the uniformity function $f:\{1\}^{*} \rightarrow \Pi$; and $e$ that maps from binary words to the multiset of unique objects in the appropriate member of $\Pi$.

Each member $\Pi_{|x|}=f\left(1^{|x|}\right)$ of $\Pi$ has one single membrane, env, that is both the cnvironment and the input membrane. On input $1^{|x|}$, the function $f$ produces a configuration graph $G_{\mathcal{T}_{, u}}$ for machine $\mathcal{T}$ on input $1^{u}$ for each $u \in\left\{1,2, \ldots, q^{\prime}(|x|)\right\}$. (Note that this is a generalization of the technique used in the proof of Lemma 4.3.) Since we have unary input words we can include the input word as part of the configuration to ensure that there is a unique input configuration for cach $G_{\mathcal{T}, u}$. 
Each of the $q^{\prime}(|x|)$ configuration graphs are converted to membrane rules and objects, using the same technique (without the second Turing machine that solves un-reachability) from the proof of Lemma 4.3, of a single membrane system $\Pi_{|x|}$. In summary, the vertices of the configuration graphs become objects in $\mathrm{I}_{|x|}$ and the edges in the graph become type (a) rules. There is a type (a) rule that maps the object encoding the accepting configuration of $\mathcal{T}$ to yes. We do not include the second Turing machine that solves un-reachability from Lemma 4.3. $\mathcal{T}$ is a logspace machine and so its configuration graph is of polynomial size, it follows that the membrane system is of polynomial size. It is relatively straightforward to verily that $f \in \mathrm{FAC}^{0}$.

The inpul encoder $\epsilon(x)$ simulates $r(x)$ to find the set of unary words $\left(u_{1}, \ldots, u_{k}\right)$, then outputs an object $c_{i . u}$ for each $u \in r(x)$, which encode the vertex of the configuration graph corresponding to the initial configurations of Turing machine $\mathcal{T}$ input $u$. Since $r \in \mathrm{FAC}^{0}$ it is not difficult to sec that $e \in \mathrm{FAC}^{0}$.

We now show that the membrane system $\Pi_{|x|}$ on input $e(x)$ accepts if $x \in L$ and otherwise rejects.

Suppose $x \in L$. This implies that at least one word in $r(x)$ is in the tally set $T$ and so $\mathcal{T}$ accepts on at least one of these inputs. The input membrane of $\mathrm{I}_{|x|}$ contains $e(x)$ which includes the object $c_{i, u}$ which encodes the configuration graph vertex that represents the initial configuration of Turing machine $\mathcal{T}$ on input $1^{u}$. In the proof of Lemma 4.3 we show how the construction of $\Pi_{|x|}$ is such that there is a sequence of rules from the input object $c_{i, u}$ to the yes object and so $\Pi_{|x|}$ on input $e(x)$ will accept.

Suppose $u \notin L$. This implies that none of the unary words $r(x)$ are in the tally set $T$ and that $\mathcal{T}$ does nol have any accepting computations on any of the words $1^{j}$ in $r(x)$. So, as in the proof of Lemma 4,3, this implies that none of the objects in $e(x)$ in the input membrane of $\Pi_{|x|}$ can evolve to the object yes in the env membrane. In this case the membrane system $\Pi_{|x|}$ on input $e(x)$ will halt without yes object; a rejecting computation for an acknowledger membrane system.

Therefore the pair of lunctions $f$ and $e$ provide a uniform family of polynomial time $\mathcal{A} \mathcal{M}^{0}{ }_{d}$ membrane systems that accept $L \in \mathrm{FAC}_{\mathrm{dtt}}^{0}$ (tallyNL).

\section{The computational power of recogniser $\geqslant 1$ membrane systems}

In this section we further investigate how the details in the definition of acceptance and rejection for recogniser membrane systems affect the computational power of uniform families of $\mathcal{A M}_{-d}^{0}$ systems.

In Section 5 we consider acknowledger membrane systems (Definition 2.9) where the absence of a yes object in the environment in the last step of any computation of a membrane system is sufficient to say that the system rejected its input. However, if we restrict to recogniser $\geqslant 1$ membrane systems, which must produce one or more yes objects in the case of an accepting computation and one or more no objects in the case of a rejecting computation (Definition 2.8) it is no longer clear if our characterisation of $\left(F A C^{0}, F A C^{0}\right)$-uniform-PMC $\mathcal{A} \mathcal{M}_{-d}^{0}$ for acknowledger systems can still hold. The best lower-bound we find is $F A C_{\mathrm{m}}^{0}$ (tallyNL), and we obtain upper-bounds of $F A C_{\mathrm{d} 11 .}^{0}$ (tallyNL) and $F A C_{\mathrm{rt1}}^{0}$ (tallyNL).

In the semi-uni lorm case the upperbound $F A C^{0}$-semi-uniform-PMC ${ }^{*} \mathcal{A M}^{0}{ }_{d} \subseteq \mathrm{NL}$ is unaflected by the restriction from acknowledger to recogniser $\geqslant 1$ membrane systems. It also turns out that these more restricted recogniser $\geqslant 1$ membrane systems have the same NL lower-bound on their power as acknowledger membrane systems (see Lemma 4.3).

Lemma 6.1. $\left(F A C^{0}, F A C^{0}\right)$-uniform-PMC $A \mathcal{M}_{-d}^{0} \subseteq \mathrm{FAC}_{\mathrm{ctt}}^{0}$ (tallyNL), for recogniser $\geqslant 1$ and recogniser membrane systems. 


\section{Proof:}

(Sketch) This prool closely lollows that of Lemma 5.2 so we just highlight the dillerences. In the proof of Lemma 5.2 the language $T$ is the sel of words $1^{(0,|x|)}$ where membrane system $f\left(1^{|x|}\right)=\prod_{|x|}$ aceepts il object $o$ in the input membrane eventually evolves to the object yes in the eno membrane. In this prool we consider the language $T^{\prime}$ that is the set ol words $l^{\langle 0,|x|\rangle}$ where in the membrane system $1 \Gamma_{|x|}$ the object $a$ does not evolve to the object no in the env membrane, in any computation. Via Lemma 2.13, this language is well-delined, i,e, can defined in terms of $o$ and $|x|$. Also, Turing machine $\mathcal{T}$ from Lemma 5.2 (that solves reachability) can be modified $[24,56]$ to give $\mathcal{T}^{\prime}$ (that solves unreachability) that accepts the language $T^{\prime}$. That is, $\mathcal{T}^{\prime}$ accepts if no object with the desired, and easy to check, property can be evolved by rule applications.

In Lemma 5.2 we defined the function $\tau \in \mathrm{FAC}^{0}$, that maps from $\{0,1\}^{*}$ to the set of tuples of unary words. Recall that $\tau(x)$ maps to a list that contains a unary string $1^{\langle o,|x|\rangle}$ for each $o$ in $e(x)$. We now prove that $\tau$ is a conjunctive reduction from $L$ to $T^{\prime}$.

Assume $x \in L$, this implies that no object in $\Pi_{|x|}$ with input $e(x)$ eventually evolves to no in the env membrane. Ilence $x \in L$ implics that $\forall w \in \tau(x), w \in T^{\prime}$.

Assume $x \notin L$, this implies that at least one object in the initial configuration of $\Pi_{|x|}(e(x))$ eventually evolves a no object in the $e n v$ membrane in each computation of $\Pi_{|x|}$. IIence $x \notin L$ implies that $\exists w \in \tau(x)$ such that $w \notin T^{\prime}$.

Lemma 6.2. $\mathrm{FAC}_{\mathrm{m}}^{0}($ tallyNL $) \subseteq\left(\mathrm{FAC}^{0}, \mathrm{FAC}^{0}\right)$-uniform-PMC $\mathcal{A} \mathcal{M}^{0}{ }_{d}$, for acknowledger and recogniser $\geqslant 1$ membranes systems.

\section{Proof:}

Let $L \in \mathrm{FAC}_{\mathrm{m}}^{0}$ (tallyNL). That is, there exists a unary language $T \subseteq\{1\}^{*}$ that is recognised by nondeterministic logspace Turing machine $\mathcal{T}$, and a function $r \in \operatorname{FAC}^{0}$ that maps $x \in\{0,1\}^{*}$ to a unary word such that $r(x) \in T$ iff $x \in L$. Let $q(|x|)=\max (\{|r(w)||w \in\{0,1\}| x \mid\})$, that is, the largest word produced by $r$ on any input of length $|x|$. Note that $q(|x|)$ is computable by $f$ since $r \in$ FAC $^{0}$.

We present an $\mathrm{FAC}^{0}$ uniform polynomial-time $\mathcal{A \mathcal { M } ^ { 0 }}{ }_{d}$ membrane family $\Pi$ that recognises $L$. The family is composed of two functions: $f:\{1\}^{*} \rightarrow \Pi$, and $e$ that maps each binary word to a multiset of objects from the appropriate member of $\Pi$.

Each member $\Pi_{|x|}=f\left(1^{|x|}\right)$ of $\Pi$ has one single membrane, env, that is both the environment and the input membrane. On input $1^{|x|}$ the lunction $f$ produces one configuration graph $C_{\mathcal{T}}, u$ lor machine $\mathcal{T}$ (that accepts $T$ ) on cach input $1^{u}, 1 \leq u \leq q(|x|)$, and one configuration graph $G_{N, u}$ for machine $N_{\mathcal{T}}$ (that accepts the compliment of $T$ ) on each input $1^{u}, 1 \leq u \leq q(|x|)$. (Note that this is a generalization of the technique used in the prool of Lemma 4.3.)

Each of the $2 q(|x|)$ configuration graphs are modified to give a set of rules and objects of a single membrane system $\mathrm{II}_{|x|}$ using the same technique as used in the proof of Lemma 4.3 . In summary, the vertices of the configuration graphs become objects in $\Pi_{|x|}$ and the edges in the graph become type (a) rules. There is a rule mapping the object encoding the accepting configuration of $\mathcal{T}$ to yes and rule mapping object encoding the accepting configuration of $N_{\mathcal{T}}$ to no. Both $\mathcal{T}$ and $N_{\mathcal{T}}$ are logspace machines and so their configuration graphs are of polynomial size and so the membrane system is of polynomial size. It is relatively straightforward to verify that $f \in \mathrm{FAC}^{0}$.

The input encoder $e(x)$ simulates $r(x)$ to find $\mathrm{l}^{u}$, then outputs two objects $c_{i, u}$ and $c_{j, u}$ which encode the vertex of the configuration graph corresponding to the initial configurations of Turing machines $\mathcal{T}$ and $N_{\mathcal{T}}$ respectively on input $1^{u}=r(x)$. Since $r \in \mathrm{FAC}^{0}$ it is not difficult to sec that $e \in \mathrm{FAC}^{0}$. 
We now show that the membrane system $\Pi_{|x|}$ on input $e(x)$ accepts if $x \in L$ and otherwise rejects.

Suppose $x \in L$. This implies that the word $r(x)=1^{u}$ is in the tally set $T$ and so at least one computation of $\mathcal{T}$ accepts $1^{u}$. It also implies that there is no computation of $N_{\mathcal{T}}$ that accepts on input $1^{u}$. The inpul membrane of $1 \mathrm{I}_{|x|}$ contains $e(x)$ which includes $c_{i, u}$ encoding the conliguration graph vertex that represents the initial configuration of Turing machine $\mathcal{T}$ on input $1^{t}$. In the proof of Lemma 4.3 we show how $\mathrm{II}_{|x|}$ has the property that there is a sequence of rules from the input object $c_{i, u}$ to the yes object and so $\mathrm{l}_{|x|}(e(x))$ will accept. Likewise there is no path from $c_{j, u}$ to no.

Suppose $u \notin L$. This implies that the word $r(u)=1^{j}$ is not in the tally set $T$ and that therefore there is no accepting configuration of $\mathcal{T}$ on input $1^{u}$, howcver, there is at least onc accepting computation of $N_{\mathcal{T}}$ on the same input. In the proof of Lemma 4.3 we show how the construction of $\Pi_{n}$ is such that there is a sequence of rules from the input object $c_{j, u}$ to the no object and so $\Pi_{n}(e(x))$ will reject. Likewise there is no path from $c_{i, u}$ to yes.

Therefore the pair of functions $f$ and $e$ provide a uniform family of polynomial time $\mathcal{A} \mathcal{M}_{-d}^{0}$ membrane systems that accept any language in $\mathrm{FAC}_{\mathrm{m}}^{0}$ (tallyNL).

\section{Open problems}

The power of recogniser membrane systems. In Sections 4 and 5 of this paper we characterise the power of acknowledger membrane systems (Definition 2.9), which are a generalisation of recogniser membrane systems. In Section 6 we give upper and lower bounds on the power of the more restricted recogniser $\geqslant 1$ membrane systems (Definition 2.8), which are closer in power to standard recogniser membrane systems. We also give upper bounds on the power of uniform and semi-uniform recogniser membrane systems (Definition 2.7), as well as showing that these classes are distinct.

However, we have not characterised the power of $\mathcal{A M}_{-d}^{0}$ recogniser membrane systems (Definition 2.7) with the kind of tight uniformity conditions used in this paper. In such systems, in an accepting computation exactly one yes object, or in a rejecting computation exactly one no object, is produced at the final step. A consequence of this is that our techniques for showing lower bounds on the power of acknowledger and recogniscr $\geqslant 1$ systems (Sections 4,5 and 6) in terms of non-deterministic logspacebounded Turing machines do not immediately carry over to recogniser systems.

As future work, we suggest that recogniser systems could be characterised via tmamhigutous nondeterministic logspace-bounded Turing machines [6]. An unambiguous machine accepts an input il' and only if it has exactly one accepting computation. Perhaps the class of problems solved by semi-uniform families of recogniser $\mathcal{A} \mathcal{M}_{-d}^{0}$ systems, i.e. FAC ${ }^{0}$-semi-unilorm-PMC* $\mathcal{A} \mathcal{M}_{-d}^{0}$, does not contain NLcomplete problems since the system cannot control how many yes objects it produces? Perhaps these semi-uniform recogniser systems can solve $s$ - $t$ connectivity for "mangrove" graphs, i.e. graphs where there is exactly one path between each pair of vertices which is contained in unambiguous logspace [6]? Formally, we conjecture that $\mathrm{FAC}^{0}$-semi-uniform-PMC ${ }^{*} \mathcal{A} M_{-d}^{0}=\mathrm{RUSPACE}(\log n)[6]$. We also conjecture that for the analogous uniform families of recogniser systems $\left(F A C^{0}, F A C^{0}\right)$-uniform-PMC $A \mathcal{M}_{-d}^{0}$ $=\mathrm{FAC}_{\mathrm{m}}^{0}(\mathrm{RUSPACE}(\log n))$. If proven, our conjectures, taken together with previous results [6], would give a restatement of the relationship between the classes L, unambiguous logspace and NL in the membrane computing model, as well as the other classes shown in Figure 1. 
Tight uniformity conditions for other classes of membrane systems. In this paper and others [33, $34,35,36,37]$, we have pul forward the idea of exploring the power of membrane systems under tight uniformity conditions. Others have sinee carried on this line of investigation [46]. Besides the main result in this paper (exhibiting systems where uniformity is a strictly weaker notion than semi-uniformity) this has led to various other characterisations of the power of a variety of classes of membrane systems and a teasing apart of their power. A number of other varieties of membrane systems (c.g. [20, 40]) characterise the complexily class $P$, but where the lower-bound actually depends on the use of $P$ unilormily, As luture work, it would be interesting to investigate these, and other, systems under suitably tight notions of uniformity or semi-uniformity.

Upper-bounding tallyNL. While we know that tallyNL $\subsetneq N L$ it would be interesting to find other classes to upper bound tallyNL. It is known that if a sparse language is complete for NL then NL $\subseteq$ DLOGTIME uniform-TC ${ }^{0}[13,23]$. Is it possible to show that tallyNL $\subseteq$ DLOGTIME uniform-TC ${ }^{0}$ ?

Classes reducible to tallyNL. We conjecture that $F A C_{\mathrm{m}}^{0}$ (tallyNL) is strictly contained in $F A C_{\mathrm{dtt}}^{0}$ (tallyNL). Giving an exact characterisation of recogniscr $\geqslant 1$ membrane systems studied in Section 6 may provide some insights into this. We also conjecture that $\mathrm{FAC}_{\mathrm{dtt}}^{0}$ (tallyNL) $\neq \mathrm{FAC}_{\mathrm{ct1} .1}^{0}$ (tallyNL). A lead to solve this may come from $K o[26]$ who showed that $P_{\text {ctt }}$ (tally) $\neq \mathrm{P}_{\mathrm{dtt}}$ (tally).

\section{Acknowledgements}

Thanks to an anonymous reviewer for a thorough reading and helpful comments. This paper appears in a special issue dedicated to Mario de Jesús Pérez-Jiménez on this 65th birthday; we thank Mario for giving us the opportunity to enjoy Sevilla and its wonderful cortados.

\section{References}

11 Adleman, L.: Molecular computation of solutions to combinatorial problems, Science, 266, 1994, 10211024.

12| Alhazov, A., Martín-Vide, C., Pan, L.: Solving a PSPACE-Complete Problem by Recognizing P Systems with Restricted Active Membranes, Fundamenta Informaticae, 58(2), 2003, 67-77.

[3] Alhazov, A., Pan, L.: Polarizationless P Systems with active membranes, Grammars, 7, 2004, 141-159.

[4] Alhazov, A., Pérez-Jimmez, M. J.: Uniform Solution to QSAI Using Polarizationless Active Membranes, in: Machines, Computations and Universality (MCU), vol. 4664 of Lecture Notes in Computer Science, Springer, $2007,122-133$.

[5] Allender, E., Koucký, M.: Amplifying lower bounds by means of self-reducibility, Journal of the ACM, 57, March 2010, 14:1-14:36.

[6] Allender, E., Lange, K.-J.: RUSPACE $(\log n) \subseteq \operatorname{DSPACE}\left(\log ^{2} n / \log \log n\right)$, Theory of Computing Systems, 31(5), 1998, 539-550.

[7] Arora, S., Barak, B.: Compututional Complexily: A Modern Approach, Cambridge University Press, 2009, ISBN 978-0-511-53381-5.

|8| Barish, R. D., Rothemund, P. W. K., Winfree, E.: Two computational primitives for algorithmic selfassembly: copying and counting, Nano Lett., 5, 2005, 2586-2592. 
[9] Barish, R. D., Schulman, R., Rothemund, P. W. K., Winfree, E.: An information-bearing seed for nucleating algorithmic sclf-asscmbly, PNAS, 106(15), 2009, 6054-6059.

|10| Book, R. V., Ko, K.-I.: On sets truth-table reducible to sparse sets, SIAM Joumal of Compuing, 17(5), 1988, 903-919.

111| Borodin, A.: On relating time and space to size and depth, SIAM Journal on Computing, 6(4), 1977, 733-744.

[12] Buhrman, H., Hemaspaandra, E., Longpre, L.: SPARSE reduces conjunctively to TALLY, SIAM Journal of Compuing, 24, June 1995, 673-681.

113| Cai, J.-Y., Sivakumar, D.: Resolution of Hartmanis' conjecture for NL-hard sparse sets, Theoretical Computer Science, 240(2), 2000, $257-269$.

[14] Chandra, A. K., Stockmeycr, L. J., Vishkin, U.: Constant depth reducibility, SIAM Journal of Compuling, 13(2), 1984, 423-439.

[15] Chen, H.-L., Doty, D., Holden, D., Thachuk, C, Woods, D., Yang, C.-T.: Fast algorithmic self-assembly of simple shapes using random agitation, DNA20: The 20ih International Con/erence on DNA Computing and Molecular Pmgramming, LNCS, Springer, Kyoto, Japan, September 2014.

[16] Condon, A., Hu, A. J., Man̆uch, J., Thachuk, C.: Less haste, less waste: on recycling and its limits in strand displacement systems, Joumal of the Royal Society-Interface focus, 2(4), 2012, 512-521.

[17] Cook, M., Soloveichik, D., Winfrec, E., Bruck, J.: Programmability of Chemical Reaction Networks, in: Algorithmic Bioprocesse's, Springer, 2009, 543-584.

|18| Dabby, N., Chen, H.-L.: Active self-assembly of simple units using an insertion primitive, SODA: Proceedings of the Twenty-fourth Annual ACM-SIAM Symposium on Discrete Algorithms, 2012.

[19] Furst, M. L., Saxc, J. B., Sipser, M.: Parity, circuits and the polynomial-time hicrarchy, The ory of Compuling Systems (formerly Mathematical Systems Theory), 17(1), 1984, 13-27.

[20] Gutiérrez-Escudero, R., Pérez-Jiménez. M. J., Rius-Font, M.: Characterizing tractability by tissue-like P systcms, in: Workshop on Membrune Compuling 10, vol. 5957 of Lecture Noles in Compuler Science, Springer, 2009, 269-181.

[21] Gutiérrez-Naranjo, M. A., Pérez-Jiménez, M. J., Riscos-Nủñez, A., Romero-Campero, F. J.: Computational efficiency of dissolution rules in membrane systems, International Joumal of Compuler Mathematics, 83(7), $2006,593-611$.

[22] Head, T., Rozenberg, G., Bladergroen, R. S., Breek, C. K. D., Lommerse, P. H. M., Spaink, H. P.: Computing with DNA by opcrating on plasmids, Biosystems, 57(2), 2000, 87-93.

[23] Hesse, W., Allender, E., Mix Barrington, D. A.: Uniform constant-depth threshold circuits for division and iterated multiplication, Journal of Computer and System Sciences, 65(4), 2002, 695-716.

124| Immerman, N.: Nondeterministic space is closed under complementation, SIAM Jounal of Computing, 17(5), 1988, 935-938.

[25] Immorman, N.: Descriptive Complexity, Springer, 1999, ISBN 0387986006.

|26| Ko, K.-I.: Distinguishing conjunctive and disjunctive reducibilities by sparse sets, Infomnation and Computation, 81(1), 1989, 62-87.

[27] Ladner, R. E., Lynch, N. A., Sclman, A. L.: A comparison of polynomial time reducibilitics, Theoretical Compuler Science, 1(2), 1975, 103-123.

[28] Lipton, R. J.: DNA solution of hard computational problems, Science, 268(5210), 1995, 542-545.

129| Liu, Q., Wang, L., Frutos, A. G., Condon, A. E., Corn, R. M., Smith, L. M.: DNA computing on surfaces, Nature, 403(6766), 2000, 175-179.

[30] Malchik, C., Winslow, A.: Tight Bounds for Active Self-Asscmbly Using an Inscrtion Primitive, ESA: The 22nd Eumpean Symposium on Algorithms, 2014, Accepted. Arxiv preprint: arXiv:1401.0359arXiv: 1401.0359 [cs.FL]. 
[31] Mauri, G., Leporati, A., Porreca, A. E., Zandron, C.: Recent complexity-theoretic results on P systems with active membranes, Journal of Logic and Compulation, 2013, (awaiting publication).

|32| Mix Barrington, D. A., Immerman, N., Straubing. H.: On uniformity within NC' ${ }^{\perp}$, Journal of Computer and System Sciences, 41(3), 1990, 274-306.

[33] Murphy, N.: Uniformity conditions for membrane systems: Uncovering complexity below 1', Ph.D. Thesis, National University of Ircland Maynooth, 2010.

[34] Murphy, N., Woods, D.: Active membrane systems without charges and using only symmetric clementary division characterise P, in: 8th International Workshop on Memhrane Computing, vol. 4860 of Lecture Notes in Computer Science, Springer, 2007, 367-384.

[35」 Murphy, N., Woods, D.: A characterisation of NL using membrane systems without charges and dissolution, in: Unconvenional Compuling, 7 th Intemational Con/erence, UC 2008, Vienna, Austria, vol. 5204 of Lecture Notes in Computer Science, Springer, 2008, 164-176.

[36] Murphy, N., Woods, D.: On acceptance conditions for membranc systems: characterisations of $L$ and $N L$, Proceedings of the Intemational Workshop on The Complexily of Simple Programs, 1, Electronic Proceedings in Theoretical Computer Science, 2009, Arxiv preprint: arXiv:0906.3327v1arXiv:0906.3327v1 $|\mathrm{cs} . \mathrm{CC}|$.

[37] Murphy, N., Woods, D.: The computational power of membrane systems under tight uniformity conditions, Natural Compuing, 10(1), 2011, 613-632.

[38] Murphy, N., Woods, D.: AND and/or OR: Uniform polynomial-size circuits, MCU: Proceedings of Machines, Computations and Universality, 128, Electronic Proceedings in Theoretical Computer Science, 2013, Arxiv preprint: arXiv: $1212.3282 \mathrm{v} 2 \mathrm{arXiv}: 1212.3282 \mathrm{v} 2[\mathrm{cs.CC}]$.

[39] Ouyang, Q., Kaplan, P. D., Liu, S., Libchaber, A.: DNA solution of the Maximal Clique Problem, Science, 278(5337), 1997, 446-449.

140| Pan. L., Pèrez-Jiménez, M. J.: Computational complexity of tissue-like P systems, Journal of Complexity, 26(3), 2010, 296-315.

[41] Papadimitriou, C. H.: Computational Complexity, Addison Wesley, 1993. ISBN 0201530821.

[42] Parberry, I.: Cirnit complexily and neural networks, MIT Press, 1994, ISBN 0-262-16148-6.

[43] Păun. G., Rozenberg, G., Salomaa, A., Eds.: The Oxford Handbook of Membrane Computing, Oxford University Press. Inc., New York, NY, USA, 2010. ISBN 0199556679, 9780199556670.

[44] Pérez-Jiméncz, M. J., Riscos-Núñcz, A., Romero-Jiméncz, A., Woods, D.: The Oxford Handbook of Memhrane systems, chapter 12: Complexity - Membrane Division, Membrane Creation, Oxford University Press, $2009,302-336$.

145| Pérez-Jiménez, M. I., Romero-Jiménez, A., Sancho-Caparrini, F.: Complexity classes in models of cellular computing with membranes, Natural Computing, 2(3), 2003, 265-285.

46| Porreca, A. E., Leporati, A.. Mauri, G.. Zandron, C.: Sublinear-space P systems with active membranes, in: Proceedings of the 13 th Intemational Conference on Membrane Computing 2012, vol. 7762 of Lecture Notes in Computer Science, Springer, 2013,342-357.

[47] Post, E. L.: Recursively cnumcrable sets of positive integers and their decision problems, Bullelin of the? American Mathematical Society, 50(5), 1944, 284-316.

|48| Păun, G.: Membrane computing, in: Fundamentals of computation theory, vol. 2751 of Lecture Notes in Computer Science, Springer, 2003, 284-295.

[49] Păun, G.: Further twenty six open problems in membranc computing, Proceedings of the Third Bruinstoming Week on Membrane Computing, Fénix Editoria, 2005.

150| Qian, L., Winfree, E.: Scaling up digital circuit computation with DNA strand displacement cascades, Science, 332(6034), 2011, 1196. 
[51] Rothemund, P. W., Winfree, E.: The program-size complexity of self-assembled squares, Proceedings of the thirty-second annual ACM symposium on Theory of compuling, ACM, 2000.

[52] Soloveichik, D., Cook, M., Winfree, L., Bruck, J.: Computation with finite stochastic chemical reaction networks, Naiural Computing, 7(4), 2008, 615-633.

[53] Soloveichik, D., Winfree, E.: The computational power of Benenson automata, Theoretical Computer Science, 344, 2005, 279-297.

154| Soloveichik, D., Winfree, E.: Complexity of self-assembled shapes, SIAM Journal of Computing, 36(6), 2007, 1544-1569.

[55] Sosik, P.: The computational power of cell division in P systems: Beating down parallel computers?, Natural Compuling, 2(3), 2003, 287-298.

[56] Szclepesćnyi, R.: The method of fored cnumeration for nondeterministic automata, Acta Informatica, 26(3), $1988,279-284$.

[57] Thachuk, C., Condon, A.: Space and energy efficient computation with DNA strand displacement systems, in: Isth International Conference on DNA Computing and Molecular Programming, vol. 7433 of Lecture Notes in Computer Science, Springcr, 2012, 135-150.

[58] Thachuk, C. J.: Space and energy efficient molecular programming and space effcient text indexing methods for sequence alignment, Ph.D. Thesis, University of British Columbia, 2013.

[59] Vollmer, H.: Introduction to Circuit Complexity: A Uniform Approach, Springer-Verlag New York, Inc., Secaucus, NI, USA, 1999, ISBN 3540643109.

[60] Woods, D., Chen, H.-L., Goodfriend, S., Dabby, N., Winfrec, E., Yin, P.: Active self-asscmbly of algorithmic shapes and patterns in polylogarithmic time, ITCS'73: Proceedings of the 4th conference on Innovations in Theoretical Computer Science, ACM, 2013, Full version: arXiv: 1301.2626arXiv: 1301 . 2626 [cs.DS]. 